\title{
DIE BETRIEBSWIRTSCHAFTLICHE BERATUNG IN DEUTSCHLAND
}

Auszug aus einem Vortrag, gehalten am 9. 11. 1956 in der Finnischen Landwirtschaftswissenschaftlichen Gesellschaft in Helsinki.

M. RoLFES

Institut für landwirtschaftliche Betriebslehre der Justus Liebig-Hochschule Giessen, Deutschland

Lange Jahrzehnte hindurch ist die landwirtschaftliche Beratung in Deutschland eine Beratung in Fragen der Produktionsmethoden gewesen, Düngung, Fütterung, Sorten- und Rassenwahl, Bodenbearbeitung, Schädlingsbekämpfung und ähnliche Dinge standen ganz im Vordergrund. Hiermit sind zunächst auch grosse Fortschritte in der Landwirtschaft erzielt worden, indem die grossen Erkenntnisse in den Naturwissenschaften für die landwirtschaftliche Praxis nutzbar gemacht wurden.

Eine stärkere Verbreitung der betriebswirtschaftlichen Beratung begann erst, nachdem die ökonomischen Wissenschaften einen gewissen Entwicklungsstand erreicht hatten und auch erst, nachdem schwerwiegende ökonomische Probleme in der praktischen Landwirtschaft ein echtes Bedürfnis nach betriebswirtschaftlicher Beratung weckten. Beide Voraussetzungen waren erst in der Zeit nach dem ersten Weltkrieg gegeben.

Bevor die weitere Entwicklung einer spezifisch betriebswirtschaftlichen Beratung verfolgt wird, wäre aber zunächst etwas über die Organisationsform der allgemeinen Beratung in Deutschland zu sagen. Von Anfang an waren und sind auch heute die Landwirtschaftsschulen zugleich die Träger der allgemeinen bäuerlichen Beratung. Bis in die neueste Zeit haben die Lehrer dieser Schulen (2-3 Diplomlandwirte und 1 Lehrerin) zugleich Unterricht (im Winter) und Beratung getrieben. Es handelt sich ganz überwiegend um allgemeine produktionstechnische Beratung, denn für Spezialfragen stehen die Experten der Landwirtschaftskammern zur Verfügung. Die Grös s e dieses Beratungsapparates ergibt sich schon aus der Zahl von 530 Schulen dieser Art bei 14.000.000 ha landwirtschaftlichen Bodens in der Bundesrepublik. Die Stä r k dieser Organisation liegt in der direkten Verbindung des Personals mit der bäuerlichen Praxis, einmal über die Schüler, ferner über verschiedene Sonderaufgaben wie Anerkennung von Saatgut, Körung von Zuchttieren und ähnliches mehr. Die S chwä ch e liegt in der Zweiteilung der Arbeit in Unterricht und Beratung, wobei der Unterricht aus verständlichen Gründen die primäre Aufgabe ist. 
Die Entwicklung einer systematischen betriebswirts chaftlichen Beratung verlief ausserhalb dieses Rahmens der offiziellen Beratung bei den Landwirtschaftsschulen. Kritische ökonomische Lagen und besondere Aufgaben waren der Anlass zu solcher Beratung. Vor allem müssen genannt werden: die Siedlerberatung im Rahmen der bäuerlichen Siedlung nach 1919, die Kreditberatung in der grossen Agrarkrise vor 25 Jahren und schliesslich die ökonomisch-technische Beratung des "Reichskuratoriums für Technik in der Landwirtschaft" im letzten Jahrzehnt vor Ausbruch des zweiten Weltkrieges.

Alle diese drei Formen der landwirtschaftlichen Beratung waren zeitlich oder räumlich beschränkt und hatten relativ enggesetzte Ziele. Aber in diesen ersten Vorstössen wurden Prinzipien entwickelt, die später - nach dem zweiten Weltkrieg - für den breiteren Aufbau dieser Art der Beratung sehr wichtig wurden. Man kann sie kurz folgendermassen umreissen:

1. Die Entscheidung über Teilmassnahmen kann immer nur getroffen werden unter voller Berücksichtigung der Wirkungen auf alle anderen Teile des Betriebes einschliesslich Arbeitswirtschaft und Gelderfolg.

2. Einzelne Betriebe (Richtbetriebe) werden besonders intensiv beraten und rechnerisch durchgearbeitet, um Unterlagen für die Beratung anderer Betriebe ähnlichen Typs zu gewinnen.

3. Die Wissenschaft wird eingeschaltet zur systematischen und kritischen Sammlung, Ordnung und Auswertung allen Materials, das aus der intensiven Einzelberatung der Richtbetriebe hervorgeht; diese wissenschaftliche Materialbearbeitung ist primär für die Förderung und Anregung der praktischen Beratungsarbeit bestimmt.

Leider kann in Deutschland kaum auf eine wirklich allgemein verbreitete Buchführung als Grundlage für betriebswirtschaftliche Beratung nicht zurückgegriffen werden, weil eine solche in den eigentlichen Bauernbetrieben aus steuerlichen Gründen selten anzutreffen ist. Soweit sie vorhanden ist, wird natürlich auch die Buchführung in der Betriebsberatung verwendet. Dabei ist es wertvoll, dass die von den Buchführungsinstituten herausgegebenen Sammelergebnisse immer mehr neben den Ziffern des Betriebsabschlusses auch vielerlei betriebswirtschaftliche Kenndaten enthalten.

Ich möchte die Entwicklung bis zum zweiten Weltkrieg kurz zusammenfassen: Die Beratung, die der grossen Masse der Landwirte zur Verfügung stand, war organisatorisch mit dem niederen landwirtschaftlichen Schulwesen eng verbunden. Sie war weitgehend auf Verbesserung der Produktionsverfahren eingestellt, obwohl es an einer Berücksichtigung betriebswirtschaftlicher Gesichtspunkte keineswegs fehlte. Diese Beratung war in der Hauptsache eine Massenberatung, die mit Vorträgen, Demonstrationen usw. zu wirken versuchte.

Dagegen waren betriebswirtschaftliche Beratungssysteme für besondere Zwecke und mit spezifischem Aktionsradius und als Individualberatung entwickelt worden: so die Siedle rberatung und die $\mathrm{Kredi}$ tberatung. Und schliesslich hat im letzten Jahrzehnt vor dem zweiten Weltkrieg die Beispielsberatung des Reichskuratoriums für Technik in der Landwirtschaft geradezu Pionierarbeit in der metho- 
dischen Entwicklung der betriebswirtschaftlichen Beratung geleistet. In allen drei Fällen - Siedlerberatung, Kreditberatung und Beratung des RKTL - stand die individuelle Beratung im Vordergrund, aber auch immer mit dem Ziel, aus dem Einzelfall das Verallgemeinerungsfähige zu erkennen.

Während des Krieges war die Landwirtschaft fest in die Zwangswirtschaft eingebaut. Aus dem Berater wurde geradezu der Kontrollbeamte des Staates. Der Bauer hatte nicht mehr die Wahl, Rat anzunehmen oder nicht; er hatte Instruktionen und Verordnungen zu befolgen. Das blieb auch zunächst nach dem Kriege so, als es notwendig war, die weitgehend zusammengebrochene landwirtschaftliche Produktion wieder aufzubauen, um die Hungersnot abzuwehren. Soweit der Bauer beraten wurde, war es immer das primäre Ziel, höhere Produktionsleistungen zu verlangen, damit die Menschen in den Städten nicht verhungerten. Etwa nach 1948 trennte sich aber die Beratung wieder von der Zwangswirtschaft. Es begann die Arbeit um den betriebswirtschaftlichen Erfolg der Bauern selbst.

In dem Wiederaufbau des betriebswirtschaftlichen Beratungswesens nach dem Kriege haben wir den Amerikanern viel zu verdanken, und zwar nicht nur materielle Hilfe, sondern auch neue Ideen. In zweierlei Richtung gaben sie uns neue Impulse:

1. in der Mitwirkung der Bauern selbst in den Beratungsorganisationen, und

2. in der Schulung der Berater unter Mitwirkung der Hochschulen.

Besonders wichtig an der Nachkriegsentwicklung ist aber dieses: Hand in Hand mit einer sehr starken E r w e i t e r u n g des ganzen landwirtschaftlichen Beratungswesens überhaupt ist es gelungen, in grosser Breite die Beratung auf eine betriebswirtschaftliche Basis zu stellen.

Zuerst etwas über die betriebswirtschaftliche Durchdring ung der allgemeinen Beratung. Man muss zwei Systeme der Beratung unterscheiden, die im Nachkriegsdeutschland dieses verwirklicht haben, aber in verschiedenen Organisationsformen. Die Verschiedenheit der Form hängt mit den regionalen Unterschieden in der Struktur unserer Landwirtschaft zusammen.

In $\mathrm{N}$ ordde u t s hland mit seinen mittel- bis grossbäuerlichen Betrieben kennen wir das System der Berat ung s ringe. Hier schliessen sich 20 bis 50 und mehr Landwirte zusammen zu einem Beratungsring und stellen sich einen Diplomlandwirt als ihren Berater an. Die Ringe werden durch Beiträge der Landwirte und darüber hinaus durch Staatszuschüsse finanziert. Die einzelnen Ringe sind zu Verbänden mit einem Zentralbüro zusammengefasst. Das Beratungssystem ist primär eine individuelle betriebswirtschaftliche Beratung auf Grund eingehender Buchführung. Darüber hinaus sind die Ergebnisse und Erfahrungen dieser Einzelberatungen eine wichtige Grundlage für die Massenberatung. Damit wird schliesslich diese Ringberatung weit über den Ring hinaus der breiten Masse der Praxis nutzbar gemacht. Durch eine sehr lebhafte Mitwirkung der Zentralbüros wird dafür gesorgt, dass der einzelne Ringberater draussen nicht in eine geistige Isolierung gerät. Ausserdem haben wir hier eine besonders starke Mitbestimmung der Landwirte selbst. 
Dieses System funktioniert in den Gebieten mit nicht zu kleinen Betrieben gut. Es lässt sich aber nicht in die typischen klein- und kleinstbäuerlichen Gebiete übertragen, die für grosse Teile von West- und Südwestdeutschland so typisch sind. Hier blieben die Landwirtschaftsschulen nach wie vor die Träger der Beratung. Nur mit wichtigen Fortschritten gegenüber früher: ein Teil des Personals dient voll und ganz der Beratung; und die Grundlage ist die intensive betriebswirtschaftliche Beratung von Einzelbetrieben als feste Basis für die darauf aufbauende Massenberatung.

In der Praxis sieht es etwa so aus: Eine Landwirtschaftsschule mit einem Arbeitsgebiet von vielleicht 40 bis 60 Gemeinden erhält zu dem bisherigen Personal (2-3 Diplomlandwirte, 1 weibliche Lehrkraft) noch 4-5 Beratungshilfskräfte. Jede von diesen übernimmt einen Bezirk mit etwa 8-12 Gemeinden. Damit steht dieser Berater oft etwa 400 oder noch mehr Betrieben gegenüber. Er kann nur versuchen, in den Gemeinden seines Bezirkes einige richtunggebende Betriebe zu gewinnen. Daher ist es das gesetzte Ziel: mindestens e in Richtbetrieb in jeder Gemeinde. Ist der Bauer eines solchen Richtbetriebes ein angesehener Mann im Kreise seiner Kollegen, so hat ein solcher Richtbetrieb eine wirklich starke Beispielswirkung, die oft ebenso sehr von der Persönlichkeit des Bauers wie von der objektiven Leistung seines beratenen Betriebes ausgeht. Dem Bauern selbst fällt also ein Teil der Aufgabe zu, die Beratung in die Breite zu tragen.

Ich habe versucht, in ganz kurzer Darstellung die Bedeutung der betriebswirtschaftlichen Komponente in der allgemeinen bäuerlichen Beratung in Deutschland zu zeigen. Im einzelnen gibt es so viele Verschiedenheiten wie wir Bundesländer in der Bundesrepublik haben. Aber das wirklich Wichtige ist, dass die betriebswirtschaftliche Denkweise, das, was der Amerikaner "Farm Unit Thinking" nennt, heute nicht mehr auf einzelne spezielle Zweige der Beratung beschränkt ist, sondern zur Grundlage des breiten, allgemeinen Beratungswesens der Länderverwaltungen geworden ist.

Es ist dabei ganz selbstverständlich, dass die Spezialberatung in Fragen der einzelnen Produktionszweige, also Düngung, Fütterung, Zucht, Rassen- und Sortenwahl usw., nach wie vor wichtig ist. Soweit es sich nicht um Fragen handelt, die nur der hochqualifizierte Spezialist beantworten kann, gehört diese Beratung selbstverständlich auch zur allgemeinen Beratungsarbeit. Darüber stehen die Spezialabteilungen der Landwirtschaftskammern und ähnliche Organisationen im Bedarfsfall zur Verfügung. Ferner wäre zu erwähnen, dass die Beratung der Frauen auf dem Lande in hauswirtschaftlichen Dingen sich immer mehr ausweitet.

Aber auch heute gibt es $\mathrm{S}$ ondera u f g b e $\mathrm{n}$ in der Beratung, die ganz spezifisch betriebswirtschaftlicher Art sind und die auch über die Arbeitskapazität der allgemeinen Beratung hinausgehen. Solche Aufgaben entstehen in Verbindung mit den staatlichen Massnahmen zur Verbesserung der Agrarstruktur unserer kleinbäuerlichen Gebiete. Hier werden in steigendem Masse von unseren Länderverwaltungen Spezialberater eingesetzt. Sie haben zwei Aufgaben: einmal die Bauern bezüglich der Flurbereinigung, der Dorfauflockerung und der eventuellen Betriebsvergrösserung betriebswirtschaftlich zu beraten, bevor in einer Gemeinde die endgültigen Massnahmen in allen Einzelheiten beschlossen werden. 
Zweitens soll nach erfolgter Durchführung der strukturverbessernden Massnahmen wieder eine besondere betriebswirtschaftliche Beratung den Bauern helfen bei der Rationalisierung ihrer umgestalteten Betriebe.

So ist die betriebswirtschaftliche Beratung heute bei uns doch in gewissem Sinne zum zentralen Kern der Beratung geworden. Damit die erweiterten Aufgaben erfüllt werden konnten, ist in allen Teilen des Landes die Zahl der Berater stark erhöht worden.

Eine wichtige Voraussetzung für den Erfolg ist aber die Schulung der Berater, sonst hilft die vermehrte Zahl garnichts. Die Schulung ist in den letzten Jahren zu einem festen Bestandteil unseres Beratungswesens geworden. Im einzelnen ist das System der Schulung von Land zu Land innerhalb der Bundesrepublik verschieden. Was aber fast überall in den Vordergrund tritt, ist die Mitwirkung der Hochschulen. So besteht an der Landwirtschaftlichen Hochschule in StuttgartHohenheim ein Institut für Beratungswesen. In $\mathrm{H}$ essen hat das Institut für landwirtschaftliche Betriebslehre der Fakultät in Giessen eine Abteilung für Wirtschaftsberatung; in Norddeutschland sind die Institute für landwirtschaftliche Betriebslehre in Göt t ingen und $\mathrm{K}$ i el stark beteiligt an der Schulung der Berater. Dies sind nur einige Beispiele.

Die Schulung wird in verschiedener Weise durchgeführt. Zum Teil werden Vortragskurse von kurzer Dauer abgehalten, zum Teil längere Ausbildungskurse durchgeführt. Ganz allgemein wird aber die Schulung als etwas immer Wiederkehrendes angesehen, das heisst, der Berater bekommt immer wieder Gelegenheit, mit seinen Kollegen zur geístigen Auffrischung an den Hochschulen zusammen zu kommen.

Recht weit entwickelt ist die Beraterschulung in Hessen, in dem Land, in dem ich arbeite. Hier gibt es eine eigene, ständige Berat e r s chule, die gemeinsam vom Landwirtschaftsministerium, den Landwirtschaftskammern und der Landwirtschaftlichen Fakultät betrieben wird. Hier werden kürzere oder längere Kurse (bis zu 2 Wochen) über betriebswirtschaftliche Beratung sowie auch über andere Teilgebiete abgehalten (Fütterung, Landtechnik, landwirtschaftliches Bauwesen, Hauswirtschaft u.a.m.). Da die Schule nicht in der Stadt, sondern mitten zwischen Bauerndörfern liegt, kann die betriebswirtschaftliche Schulung zum Teil direkt in die landwirtschaftlichen Betriebe hineingelegt werden. Das System dieser betriebswirtschaftlichen Schulung besteht im Besuch eines landwirtschaftlichen Betriebes, Erfassung seiner jetzigen betriebswirtschaftlichen Verhältnisse, B e urteilung dieser Verhältnisse insgesamt und im Detail, dann Aufstellung eines Beratungsieles auch wieder insgesamt und in Teilabschnitten, Kalkulierung des voraussichtlichen Erfolges, aber auch eingehende Erörterung etwaiger Finanzierungsprobleme, oft unter Mitwirkung des Bauern selbst. Hier scheint mir die Einbeziehung des praktischen Beispiels in die grundsätzlichsystematische Schulung ein recht interessantes Experiment zu sein.

Ich habe nun versucht, Ihnen ein Bild der betriebswirtschaftlichen Beratung im heutigen Westdeutschland zu geben. Ich, als Vertreter der landwirtschaftlichen 
Betriebslehre, freue mich, dass wir einiges erreicht haben. Aber ich weiss, dass wir das Erreichte nicht allein uns selber, sondern in sehr vielen Punkten der internationalen Zusammenarbeit und dem Gedankenaustausch der Experten verdanken. Wir in Westdeutschland haben viele Anregungen erhalten, in besonderem Masse von Amerika, aber auch von der Schweiz, von Holland, von den nordischen Ländern und anderen Nachbarvölkern.

SELOSTUS:

MAATALOUDEN LIIKETALOUSNEUVONTA SAKSASSA

M. ROLFES

Institut für landwirtschaftliche Betriebslehre der Justus Liebig-Hochschule Giessen.

Artikkelissa käsitellään maatalouden taloudellista neuvontatyötä Saksassa Maataloustieteellisessä Seurassa, Helsingissä, 9. 11. 1956 pidetyn esitelmän selostuksena. 